Published in final edited form as:

J Am Soc Hypertens. 2014 June ; 8(6): 414-421. doi:10.1016/j.jash.2014.03.330.

\title{
Arterial Stiffness is Associated with Increase in Blood Pressure Over Time in Treated Hypertensives
}

\author{
T Coutinho, MD*, KR Bailey, $\mathrm{PhD}^{\dagger}, \mathrm{ST}$ Turner, MD, and IJ Kullo, MDף \\ *Department of Internal Medicine, Division of Cardiology, University of Ottawa Heart Institute \\ ${ }^{\circ}$ Department of Medicine, Division of Nephrology and Hypertension, Mayo Clinic, Rochester, MN \\ tDepartment of Biomedical Statistics and Informatics, Mayo Clinic, Rochester, MN \\ IDivision of Cardiovascular Diseases, Mayo Clinic, Rochester, MN
}

\begin{abstract}
Background-Arterial stiffness is associated with incident hypertension. We hypothesized that arterial stiffness would predict increases in systolic (SBP), mean (MAP) and pulse pressure (PP) over time in treated hypertensives.
\end{abstract}

Methods-Blood pressure (BP) was measured a mean of $8.5 \pm 0.9$ years apart in 414 nonHispanic white hypertensives (mean age $60 \pm 8$ years, $55 \%$ women). The average of 3 supine right brachial BPs was recorded. Measures of arterial stiffness including carotid-femoral pulse wave velocity (cfPWV), aortic augmentation index (AIx) and central pulse pressure (CPP) were obtained at baseline by applanation tonometry. We performed stepwise multivariable linear regression analyses adjusting for potential confounders to assess the associations of arterial stiffness parameters with BP changes over time.

Results-Systolic, mean and pulse pressure increased in $80 \%$ of participants. After adjustment for the covariates listed above, cfPWV was significantly associated with increases in SBP $(\beta \pm \mathrm{SE}$ : $0.71 \pm 0.31)$ and $\mathrm{PP}(\beta \pm \mathrm{SE}: 1.09 \pm 0.27)$; AIx was associated with increases in SBP $(\beta \pm \mathrm{SE}$ : $0.23 \pm 0.10)$ and MAP $(\beta \pm$ SE: $0.27 \pm 0.07)$; and CPP was associated with increases in SBP $(\beta \pm$ SE: $0.44 \pm 0.07)$, MAP $(\beta \pm$ SE: $0.24 \pm 0.05)$ and PP $(\beta \pm$ SE: $0.42 \pm 0.06)$ over time ( $P \unlhd) .02$ for all).

Conclusions-Baseline arterial stiffness measures were associated with longitudinal increases in SBP, MAP and PP in treated hypertensives.

\section{Keywords}

hypertension; pulse wave velocity; pulse pressure; augmentation index

(C) 2014 American Society of Hypertension. Published by Elsevier Inc. All rights reserved.

Address for correspondence: Iftikhar J. Kullo, MD, Mayo Clinic, Division of Cardiovascular Diseases, 200 First Street SW, Rochester, MN 55905, FAX: 507-266-1702; TEL: 507-284-9049, kullo.iftikhar@ mayo.edu.

Publisher's Disclaimer: This is a PDF file of an unedited manuscript that has been accepted for publication. As a service to our customers we are providing this early version of the manuscript. The manuscript will undergo copyediting, typesetting, and review of the resulting proof before it is published in its final citable form. Please note that during the production process errors may be discovered which could affect the content, and all legal disclaimers that apply to the journal pertain.

Conflicts of Interest: None of the authors have any conflicts of interest to disclose. 


\section{Introduction}

Hypertension is a major cause of morbidity in the United States, being associated with coronary artery disease,(1) stroke, (2) renal disease(3) and heart failure.(4) Hypertension affects 1 in 3 American adults, (5) and only $48 \%$ of those aware of their condition achieve optimal blood pressure (BP) control.(5) Even small increments in BP are clinically relevant, as a $2 \mathrm{~mm} \mathrm{Hg}$ increase in systolic BP has been shown to be associated with a 7\% increase in mortality from coronary artery disease and $10 \%$ increase in stroke mortality. (6) Moreover, the number of deaths attributable to hypertension increased by nearly 50\% between 1998 and 2008,(5) highlighting the need for better strategies to identify and treat those at greater risk for worsening of hypertension and adverse outcomes.

In the past decade attention has focused on the associations of arterial stiffness with cardiovascular risk factors and adverse outcomes. The aorta not only functions as a conduit of blood, but also buffers the pulsatile energy generated by the heart with each cardiac cycle, thereby decreasing afterload and stroke work, and preventing the delivery of deleterious, highly pulsatile energy to the end-organs. As the aorta stiffens, there are greater swings in BP, pulse pressure increases, cardiac function is impaired and end-organ damage ensues.(7) As a result, arterial stiffness is independently associated with adverse cardiovascular events (8) and mortality,(9-11) and the European Societies of Cardiology and Hypertension have recommended assessment of arterial stiffness in all hypertensives for whom the technique is available.(12)

Given the deleterious effects of hypertension on cardiovascular health and survival, there is a need for markers that identify hypertensive individuals whose BPs are more likely to increase over time, so they can be treated more aggressively to reduce adverse outcomes. Greater aortic stiffness has been shown to be associated with incident hypertension and increases in BP over time in the general population. (13) Whether measures of arterial stiffness predict longitudinal changes in BP in treated hypertensive individuals remains unknown. To address this gap in knowledge, we studied a cohort of hypertensives from the general population to determine whether measures of arterial stiffness (carotid-femoral pulse wave velocity, aortic augmentation index and central pulse pressure) predict longitudinal changes in systolic, mean and pulse pressure.

\section{Methods}

\section{Study participants}

The study participants consisted of 414 hypertensive non-Hispanic white participants from the Genetic Epidemiology Network of Arteriopathy (GENOA) study $(14,15)$ who underwent measurement of arterial stiffness on 2 separate occasions (between January 2003 and December 2008, and between October 2009 and December 2011). The GENOA study is community-based study aimed at identifying genetic variants influencing BP levels and the development of target-organ damage due to hypertension. Participants belong to sibships with at least 2 family members diagnosed with essential hypertension before the age of 60 years. The diagnosis of hypertension was established based on a prior diagnosis of hypertension and/or current treatment with medications for hypertension. The study was 
approved by the Mayo Clinic's Institutional Review Board and participants gave informed consent.

\section{Assessment of BP and baseline characteristics}

On the day of the study, participants met with the study coordinator and completed a comprehensive questionnaire that included demographic, social, family and medical information. Brachial systolic (SBP) and diastolic blood pressures (DBP) were measured in the supine position by trained technicians 3 consecutive times with a random-zero sphygmomanometer, by auscultating at 2-min intervals, and their average was used for analyses. Mean brachial arterial pressure (MAP) was calculated as $[(2 * \mathrm{DBP})+\mathrm{SBP}] / 3$. Brachial pulse pressure (PP) was calculated as brachial SBP - DBP.

A blood specimen was collected, and serum creatinine and glucose were measured by standard enzymatic methods. Glomerular filtration rate (GFR) was estimated based on the Modification of Diet in Renal Disease equation.(16) Diabetes was considered present if a subject was being treated with insulin or oral agents, or had a fasting glucose level $\geq 7.0$ $\mathrm{mmol} / \mathrm{L}$ (>126 mg/dL). 'Ever' smoking was defined as having smoked more than 100 cigarettes in the past. Weight (in $\mathrm{kg}$ ) was measured by an electronic scale, height (in meters) by a stadiometer, and body mass index (BMI) was calculated in units of $\mathrm{kg} / \mathrm{m}^{2}$.

\section{Arterial tonometry}

We assessed 3 measures of arterial stiffness: carotid-femoral pulse wave velocity (cfPWV), considered the gold standard measure of aortic stiffness, aortic augmentation index (AIx), a measure of arterial wave reflection, and central pulse pressure (CPP), a global measure of arterial stiffness. Participants were asked to fast for $12 \mathrm{~h}$ and withhold vasoactive medications, alcohol and caffeine $24 \mathrm{~h}$ prior to the study visit. Arterial tonometry of the right carotid, radial and femoral arteries was performed at the time of the initial study visit using the Sphygmocor apparatus (AtCor Medical, Sydney, Australia) with simultaneous ECG recording as previously described.(17) Transit distances were obtained with the subtraction method from body surface measurements from the carotid sampling site to the manubrium sternum and from the manubrium sternum to the femoral artery. The time (t) between the onset of carotid and femoral waveforms was determined as the mean of 10 consecutive cardiac cycles. cfPWV was calculated from the distance between measurement points (D) and the measured time delay (t) as follows: $c f P W V=D / t(m / s)$, where $D$ is distance in meters and $t$ is the time interval in seconds. An aortic pressure waveform was derived from the radial artery waveforms using a generalized transfer function.(18) From the derived aortic pressure waveform, CPP was calculated as the difference between central SBP and DBP. Aortic augmentation pressure was calculated as the difference between the first and second systolic peaks of the ascending aortic waveform, and AIx was expressed as a percentage of the CPP.

\section{Statistical analyses}

Continuous variables are reported as mean \pm standard deviation (SD). Differences in BP parameters between the $1^{\text {st }}$ and $2^{\text {nd }}$ study visits were compared using a paired t-test. Categorical variables were reported as number (n) and percentages of the total (\%). 
Changes in BP (SBP, DBP, MAP and PP) over time were determined by calculating the difference between absolute values of BP components (BP at $2^{\text {nd }}$ visit $-\mathrm{BP}$ at $1^{\text {st }}$ visit). We developed multivariable linear regression models to assess the associations of baseline cfPWV, AIx and CPP with longitudinal changes in BP components. To account for relatedness among the participants, all regression models were performed using generalized estimating equations. Covariates considered for inclusion in the multivariate models were: age, sex, time interval between the 2 visits, baseline SBP and DBP, history of diabetes, smoking, myocardial infarction or stroke, BMI, GFR, use of statins and difference in the number of anti-hypertensive medications between $2^{\text {nd }}$ and $1^{\text {st }}$ visits. Only covariates significantly associated $(P \unlhd 0.05)$ with the dependent variable were included in the final models, but age and sex were forced into all models. To determine whether age and sex modified the associations between arterial stiffness and hypertension progression, interaction terms for age, sex and each arterial stiffness variable were added to the models.

Lastly, we stratified the participants by quartiles of cfPWV, AIx and CPP and assessed the associations of increasing quartiles with "worsening of hypertension" during follow-up using multivariable logistic regression models adjusted in stepwise fashion as in the linear regression models above. "Worsening of hypertension" was defined as a difference between absolute BP values between the $2^{\text {nd }}$ and $1^{\text {st }}$ visits $>0$.

Statistical analyses were performed with SPSS vs. 21 (IBM Corp., Armonk, NY), and a $P$ value $₫ \mathbf{} 0.05$ was considered to be statistically significant.

\section{Results}

The mean $\pm \mathrm{SD}$ age at the time of the first visit was $60 \pm 8$ years, $55 \%$ were women, and $14 \%$ were diabetic. The average interval between the 2 study visits was $8.5 \pm 0.9$ years. More participants were being treated with anti-hypertensives at the time of the $2^{\text {nd }}$ visit $(96 \%)$ than at the first visit (91\%), and the average number of anti-hypertensives per participant increased over time $\left(1.5 \pm 0.8\right.$ at the $1^{\text {st }}$ visit, $2.1 \pm 1.0$ at the $2^{\text {nd }}$ visit, $\left.P<0.0001\right)$. Despite the increase in the number of hypertension drugs, average SBP, MAP and PP increased over time (Table 2).

Linear regression assumptions were tested and satisfied. Independent predictors of increases in SBP, DBP, MAP and PP over time are depicted in Table 3. These variables were included in the final multivariable models. The results of the multivariable linear regression models are outlined in Table 4. Baseline cfPWV was directly associated with changes in SBP and $\mathrm{PP}$, and inversely associated with changes in DBP, but not associated with changes in MAP. Baseline AIx was directly associated with changes in SBP, DBP and MAP, but not with changes in PP. Baseline CPP was directly associated with changes in SBP, MAP and PP, but not with changes in DBP. When we calculated individual $Z$ scores for each BP component during both visits, and repeated the analyses utilizing a difference in $\mathrm{Z}$ scores as the dependent variable rather than absolute BP difference, inferences remained unchanged (analyses not shown). When we repeated the models adjusting for anti-hypertensive drug classes (diuretics, beta blockers, calcium channel blockers and inhibitors of the renin- 
angiotensin-aldosterone system) instead of difference in the number of anti-hypertensive drugs, the results remained unchanged (analyses not shown).

Interaction term analyses showed that age was a significant effect modifier of the associations of AIx and CPP with longitudinal changes in MAP. When we stratified the sample into age < and $\nsucceq 65$ years, we found that AIx and CPP were significantly associated with increases in MAP among those younger than 65 years ( $\beta \pm$ SE: $0.35 \pm 0.08$ and $0.23 \pm 0.06$, respectively, $P<0.0001$ for both) but not in those $>65$ years $(\beta \pm$ SE: $0.04 \pm 0.10$, $P=0.69$ and $0.18 \pm 0.09, \mathrm{P}=0.052$, respectively). We did not find significant associations between sex and arterial stiffness measures in the prediction of BP change over time.

The cutoff values for the $2^{\text {nd }}, 3^{\text {rd }}$ and $4^{\text {th }}$ quartiles of cfPWV were $8.2 \mathrm{~m} / \mathrm{s}, 9.4 \mathrm{~m} / \mathrm{s}$ and 11.0 $\mathrm{m} / \mathrm{s}$, respectively; cutoff values for the $2^{\text {nd }}, 3^{\text {rd }}$ and $4^{\text {th }}$ AIx quartiles were $27 \%, 33 \%$ and $39 \%$, respectively; cutoff values for the $2^{\text {nd }}, 3^{\text {rd }}$ and $4^{\text {th }}$ quartiles of CPP were $37 \mathrm{mmHg}, 45$ $\mathrm{mmHg}$ and $55 \mathrm{mmHg}$, respectively. Independent predictors of worsening of SBP, DBP, MAP and PP are listed in table 5. The results of the final multivariable logistic regression models are shown in Figure 1. Increasing quartiles of cfPWV were associated with worsening of SBP and PP; while increasing quartiles of AIx were associated with worsening of SBP and MAP, and increasing quartiles of CPP were associated with worsening of SBP, MAP and PP. As an example, a participant with a baseline cfPWV greater than $11 \mathrm{~m} / \mathrm{s}$ was 2.5 times more likely to experience an increase in SBP and 3.6 times more likely to experience an increase in PP during follow-up than a participant whose cfPWV at baseline was lower than $8.2 \mathrm{~m} / \mathrm{s}$. Inferences were similar when worsening of hypertension was defined as a difference in BP value $\mathrm{Z}$ scores between the $2^{\text {nd }}$ and $1^{\text {st }}$ visits $>0$ (analyses not shown).

\section{Discussion}

In a community-based cohort of hypertensive individuals, most of whom were receiving anti-hypertensive therapy ( $91 \%$ and $96 \%$ at the $1^{\text {st }}$ and $2^{\text {nd }}$ visits, respectively), we investigated the associations of measures of arterial stiffness with changes in BP over a mean period of 8.5 years, and found that arterial stiffness was associated with longitudinal increases in SBP, MAP and PP, and decrease in DBP. Our findings are relevant for clinical practice as they highlight arterial stiffness as a possible key factor in the pathophysiology of progression of hypertension, an important health burden(5) with associated high morbidity, mortality $(1,2,5,6)$ and health care costs. (5) Notably, few of the clinical parameters were associated with hypertension progression, and baseline SBP and DBP were actually inversely associated with BP increases over time, which may have occurred due to regression towards the mean. Thus, our data show that relying solely on the clinical variables is imperfect when trying to predict the risk of hypertension progression. Noninvasive markers that identify hypertensive individuals at risk for worsening of their disease over time will be valuable for risk stratification and to individualize therapies that may help prevent the adverse consequences of hypertension. Based on our findings, measures of arterial stiffness, which can be relatively inexpensively and non-invasively obtained in the office, are candidate markers for such approach. 
In addition, we found that the associations of AIx and CPP with MAP was only present in younger $(<65$ years) subjects. Previous studies in the general population have shown that AIx increases with age until approximately age 60 , when it then plateaus and then subsequently drops slightly, ${ }^{25}$ due to predominant aortic stiffening and decrease in wave reflections with aging. As such, AIx/ wave reflections also have a greater contribution to CPP in younger than in older individuals. ${ }^{25}$ In addition, while MAP increases with aging in those younger than age 55, the elderly predominantly experience longitudinal increases in PP with only little changes in MAP. ${ }^{25}$ These changes in BP hemodynamics with aging likely explain the exclusive association of AIx and CPP with MAP in younger subjects.

Another interesting observation was that the association of brachial SBP with PP worsening was inverse, and that of DBP with PP worsening was direct, suggesting that baseline brachial PP was inversely associated with PP increase; while the baseline CPP was directly associated with PP increase. These findings are consistent with our finding that aortic stiffness is associated with hypertension progression, and can be explained on the basis of peripheral pulse pressure amplification: In younger and healthy subjects, there is greater amplification of pulse pressure from the aorta to peripheral arteries (Brachial PP/ central $\mathrm{PP}>1.0$ ). With aging and aortic stiffening, pressure amplification is reduced, with central PP approximating or even surpassing peripheral PP. As such, a greater CPP in respect to brachial PP denotes greater arterial stiffness, and based on the findings from our study, is a better predictor of $\mathrm{PP}$ worsening over time.

In normotensive individuals from the general population, measures of arterial stiffness are associated with increases in BP over time, and with incident hypertension. Liao et al(19) studied nearly 7000 normotensive individuals from the ARIC study and found that carotid stiffness predicted incidence of hypertension after 6 years of follow-up. In 1759 individuals from the Framingham Offspring Cohort,(13) $40 \%$ of whom were hypertensive, arterial stiffness (assessed by cfPWV, AIx and forward pressure wave amplitude) was associated with higher BP at follow-up, after a mean of 6.5 years. The association of these measures with longitudinal changes in BP was not assessed, however. In a cohort of 449 normotensive $(n=306)$ and untreated hypertensive $(n=143)$ individuals, Najjar et al (20) also found that cfPWV predicted increases in BP over time as well as incident hypertension. In addition, in 475 Japanese individuals with high-normal BP, (21) and in 2512 Greek normotensives, arterial stiffness (assessed by the brachial ankle pulse wave velocity in the former study, and by echocardiography in the latter) also predicted future development of hypertension. The converse association, however, is controversial. While AlGhatrif et al demonstrated that higher baseline BP was associated with faster increases in aPWV over time, (22) Kaess et al showed that baseline BP was not associated with higher aPWV after 7 years of follow-up.

To the best of our knowledge, our study is the first to prospectively assess whether arterial stiffness is associated with longitudinal increases in BP in treated hypertensives. In multivariable models we found that there were few significant predictors of longitudinal BP changes and worsening of hypertension in treated hypertensives (baseline SBP, DBP and history of myocardial infarction, although eGFR and history of smoking also predicted changes in DBP, and the difference in the number of anti-hypertensives between the 2 visits 
also predicted changes in MAP), limiting the ability to identify individuals at risk for hypertension progression based on clinical and laboratory characteristics alone, and underscoring the usefulness of arterial stiffness measures in this setting. Our findings support the use of arterial tonometry in hypertensives to identify those at greatest risk of hypertension progression and its related adverse outcomes.

In contrast to previous studies of hypertensives that showed that SBP and DBP decreased over time, (23-26) we found that BP measures increased in up to 80\% (for increases in PP) of participants in our study. BP can be divided into a steady component, MAP, and a pulsatile component, PP. Although both MAP and PP increased on average, PP increased more so than MAP $(+13 \pm 3 \mathrm{mmHg}$ compared to $+3 \pm 1 \mathrm{mmHg}$ in 8.5 years). This is consistent with previous studies in the general population, $(13,27)$ and suggests that progressive stiffening of the aorta plays a major role in the increase in BP over time, since a compliant aorta is necessary to maintain a low PP for a given stroke volume. This further corroborates our findings and highlights arterial stiffness as a key determinant of the increases in BP in hypertensive individuals despite treatment.

We have previously shown that women have greater proximal aortic stiffness and peripheral wave reflection, and consequently, higher central pulse pressure than men.(28) However, in the present study, worsening of hypertension was not more common in women, and the associations of arterial stiffness measures with changes in BP over time did not differ based on sex. Thus, although arterial stiffness seems to differentially affect cardiac function and ventricular-arterial coupling in women, it is associated with future worsening of hypertension in both sexes. We did not measure proximal aortic stiffness (aortic characteristic impedance) at baseline, and in the context of our previous findings,(28) it is possible that an association of aortic characteristic impedance with worsening of hypertension may differ in men and women, which remains amenable for future studies.

\section{Strengths and Limitations}

The main strengths of our study are its community-based and prospective nature, the relatively long follow-up period, and the focus on treated hypertensives. A limitation is the restriction to non-Hispanic whites, and therefore further studies are necessary in hypertensive individuals of other ethnic groups. In addition, the cohort may not have been sufficiently powered to detect weaker predictors of BP change over time. Further, the BP recorded during the study visits may not accurately reflect BP oscillations during the day. However, our methods for assessing BP were standardized, performed in a controlled research setting, and similar to the methods used in previous studies of this topic. Lastly, we did not have data on long-term alcohol, caffeine and dairy consumption, and although we had comprehensive data on anti-hypertensive medication use, we cannot comment on changes in the doses of these medications over time.

\section{Conclusions}

In a community-based cohort of treated hypertensive individuals, measures of arterial stiffness (cfPWV, AIx and CPP) independently predicted longitudinal increases in BP. These results highlight measurement of arterial stiffness as a potential tool to identify 
hypertensive individuals at greater risk for worsening of their hypertension and who may be candidates for more aggressive risk management strategies. Arterial tonometry is noninvasive, relatively inexpensive, and can be performed in the office setting. In addition, our findings motivate further studies of arterial stiffness as a therapeutic target to prevent worsening of hypertension, and ultimately, end-organ damage and cardiovascular events.

\section{Acknowledgments}

None

Sources of Funding

This work was supported by Grants HL89354 and M01 RR00585 from the National Institutes of Health.

\section{References}

1. Kannel WB, Schwartz MJ, McNamara PM. Blood pressure and risk of coronary heart disease: the Framingham study. Dis Chest. 1969; 56:43-52. [PubMed: 5789839]

2. Kannel WB, Wolf PA, Verter J, McNamara PM. Epidemiologic assessment of the role of blood pressure in stroke. The Framingham study. J Am Med Associat. 1970; 214:301-10.

3. Klahr S, Schreiner G, Ichikawa I. The progression of renal disease. New Engl J Med. 1988; 318:1657-66. [PubMed: 3287163]

4. Dunlay SM, Roger VL, Weston SA, Jiang R, Redfield MM. Longitudinal changes in ejection fraction in heart failure patients with preserved and reduced ejection fraction. Circ Heart Fail. 2012; 5:720-6. [PubMed: 22936826]

5. Roger VL, Go AS, Lloyd-Jones DM, et al. Heart disease and stroke statistics-2012 update: a report from the American Heart Association. Circulation. 2012; 125:e2-e220. [PubMed: 22179539]

6. Lewington S, Clarke R, Qizilbash N, Peto R, Collins R. Age-specific relevance of usual blood pressure to vascular mortality: a meta-analysis of individual data for one million adults in 61 prospective studies. Lancet. 2002; 360:1903-13. [PubMed: 12493255]

7. Coutinho T, Turner ST, Kullo IJ. Aortic pulse wave velocity is associated with measures of subclinical target organ damage. JACC Cardiovasc Imaging. 2011; 4:754-61. [PubMed: 21757166]

8. Mitchell GF, Hwang SJ, Vasan RS, et al. Arterial stiffness and cardiovascular events: the Framingham Heart Study. Circulation. 2010; 121:505-11. [PubMed: 20083680]

9. Vlachopoulos C, Aznaouridis K, Stefanadis C. Prediction of cardiovascular events and all-cause mortality with arterial stiffness: a systematic review and meta-analysis. J Am Coll Cardiol. 2010; 55:1318-27. [PubMed: 20338492]

10. Vlachopoulos C, Aznaouridis K, O'Rourke MF, Safar ME, Baou K, Stefanadis C. Prediction of cardiovascular events and all-cause mortality with central haemodynamics: a systematic review and meta-analysis. Eur Heart J. 2010; 31:1865-71. [PubMed: 20197424]

11. Ben-Shlomo Y, Spears M, Boustred C, et al. Aortic pulse wave velocity improves cardiovascular event prediction: an individual participant meta-analysis of prospective observational data from 17,635 subjects. J Am Coll Cardiol. 2014; 63:636-46. [PubMed: 24239664]

12. Mancia G, De Backer G, Dominiczak A, et al. 2007 Guidelines for the management of arterial hypertension: The Task Force for the Management of Arterial Hypertension of the European Society of Hypertension (ESH) and of the European Society of Cardiology (ESC). Eur Heart J. 2007; 28:1462-536. [PubMed: 17562668]

13. Kaess BM, Rong J, Larson MG, et al. Aortic stiffness, blood pressure progression, and incident hypertension. J Am Med Associat. 2012; 308:875-81.

14. Kardia SL, Rozek LS, Krushkal J, et al. Genome-wide linkage analyses for hypertension genes in two ethnically and geographically diverse populations. Am J Hypertens. 2003; 16:154-7. [PubMed: 12559685] 
15. Coutinho Tde A, Turner ST, Peyser PA, Bielak LF, Sheedy PF 2nd, Kullo IJ. Associations of serum uric acid with markers of inflammation, metabolic syndrome, and subclinical coronary atherosclerosis. Am J Hypertens. 2007; 20:83-9. [PubMed: 17198917]

16. Levey AS, Bosch JP, Lewis JB, Greene T, Rogers N, Roth D. A more accurate method to estimate glomerular filtration rate from serum creatinine: a new prediction equation. Modification of Diet in Renal Disease Study Group. Ann Intern Med. 1999; 130:461-70. [PubMed: 10075613]

17. Kullo IJ, Bielak LF, Turner ST, Sheedy PF 2nd, Peyser PA. Aortic pulse wave velocity is associated with the presence and quantity of coronary artery calcium: a community-based study. Hypertension. 2006; 47:174-9. [PubMed: 16380523]

18. Gallagher D, Adji A, O'Rourke MF. Validation of the transfer function technique for generating central from peripheral upper limb pressure waveform. Am J Hypertens. 2004; 17:1059-67. [PubMed: 15533735]

19. Liao D, Arnett DK, Tyroler HA, et al. Arterial stiffness and the development of hypertension. The ARIC study. Hypertension. 1999; 34:201-6. [PubMed: 10454441]

20. Najjar SS, Scuteri A, Shetty V, et al. Pulse wave velocity is an independent predictor of the longitudinal increase in systolic blood pressure and of incident hypertension in the Baltimore Longitudinal Study of Aging. J Am Coll Cardiol. 2008; 51:1377-83. [PubMed: 18387440]

21. Yambe M, Tomiyama H, Yamada J, et al. Arterial stiffness and progression to hypertension in Japanese male subjects with high normal blood pressure. J Hypertens. 2007; 25:87-93. [PubMed: 17143178]

22. AlGhatrif M, Strait JB, Morrell CH, et al. Longitudinal trajectories of arterial stiffness and the role of blood pressure: the Baltimore Longitudinal Study of Aging. Hypertension. 2013; 62:934-41. [PubMed: 24001897]

23. Egan BM, Zhao Y, Axon RN. US trends in prevalence, awareness, treatment, and control of hypertension, 1988-2008. J Am Med Associat. 2010; 303:2043-50.

24. Luepker RV, Steffen LM, Jacobs DR Jr, Zhou X, Blackburn H. Trends in blood pressure and hypertension detection, treatment, and control 1980 to 2009: the Minnesota heart survey. Circulation. 2012; 126:1852-7. [PubMed: 22962433]

25. Antikainen RL, Kastarinen MJ, Jousilahti P, et al. Despite evidence-based guidelines, systolic blood pressure remains inadequately controlled in older hypertensive adults. J Hum Hypertens. 2010; 24:439-46. [PubMed: 19890369]

26. Andersen UO, Jensen GB. Trends and determinant factors in hypertension control in a population study with 25 years of follow-up. J Hypertens. 2010; 28:1091-6. [PubMed: 20186098]

27. Mitchell GF, Wang N, Palmisano JN, et al. Hemodynamic correlates of blood pressure across the adult age spectrum: noninvasive evaluation in the Framingham Heart Study. Circulation. 2010; 122:1379-86. [PubMed: 20855656]

28. Coutinho T, Borlaug BA, Pellikka PA, Turner ST, Kullo IJ. Sex differences in arterial stiffness and ventricular-arterial interactions. J Am Coll Cardiol. 2013; 61:96-103. [PubMed: 23122799] 

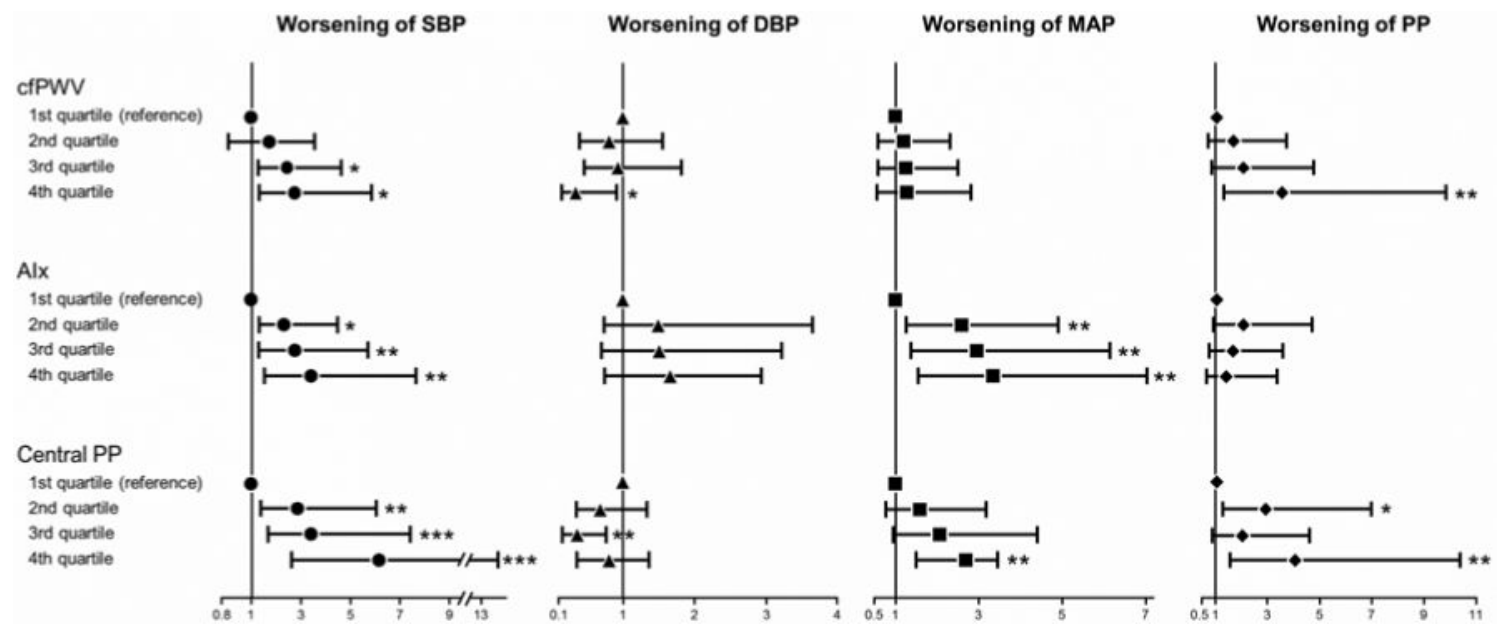

Figure 1. Associations of quartiles of arterial stiffness measures with worsening of hypertension The figure depicts the point estimates of odds ratio and respective $95 \%$ confidence intervals for the associations of each baseline measure of arterial stiffness with 'worsening' of SBP, DBP, MAP and PP, which was defined as a difference in each BP value between $2^{\text {nd }}$ and $1^{\text {st }}$ visits $>0$.

$* P \unlhd 0.05 * * P \unlhd 0.01 * * * P \unlhd 0.001$ 


\section{Table 1}

Baseline characteristics of the participants at the time of the first visit

\begin{tabular}{|l|l|}
\hline Variable $(\mathbf{n}=\mathbf{4 1 4})$ & Mean \pm SD or n (\%) \\
\hline Age, years & $60 \pm 8$ \\
\hline Women, $\mathrm{n}(\%)$ & $227(55 \%)$ \\
\hline Use of anti-hypertensive medications, $\mathrm{n}(\%)$ & $378(91 \%)$ \\
\hline Use of beta- blockers, $\mathrm{n}(\%)$ & $175(42 \%)$ \\
\hline Use of calcium channel blockers, $\mathrm{n}(\%)$ & $67(16 \%)$ \\
\hline Use of diuretics, $\mathrm{n}(\%)$ & $203(49 \%)$ \\
\hline Use of RAAS inhibitors, $\mathrm{n}(\%)$ & $187(45 \%)$ \\
\hline Use of statins, $\mathrm{n}(\%)$ & $154(37 \%)$ \\
\hline History of ever smoking, $\mathrm{n}(\%)$ & $196(47 \%)$ \\
\hline History of diabetes, $\mathrm{n}(\%)$ & $58(14 \%)$ \\
\hline History of myocardial infarction, $\mathrm{n}(\%)$ & $27(7 \%)$ \\
\hline History of stroke, $\mathrm{n}(\%)$ & $10(2.1 \%)$ \\
\hline Body mass index, $\mathrm{kg} / \mathrm{m}^{2}$ & $31.4 \pm 5.7$ \\
\hline Waist circumference, cm & $103.0 \pm 15.8$ \\
\hline Serum creatinine, $\mu$ mol/ $\mathrm{L}{ }^{*}$ & $79.6 \pm 21.2$ \\
\hline $\begin{array}{l}\text { Estimated glomerular filtration rate, } \\
(\mathrm{mL} / \mathrm{s} / 1.73 \mathrm{~m}) \dagger\end{array}$ & $1.1 \pm 0.2$ \\
\hline cfPWV, m/s & $9.7 \pm 2.6$ \\
\hline AIx $(\%)$ & $32.3 \pm 10.0$ \\
\hline Central pulse pressure, mmHg & $46 \pm 13$ \\
\hline
\end{tabular}

AIx: aortic augmentation index. cfPWV: carotid-femoral pulse wave velocity. RAAS: renin-angiotensin-aldosterone system.

* To convert serum creatinine to $\mathrm{mg} / \mathrm{dL}$, divide the value by 88.4 .

${ }^{\dagger}$ To convert estimated glomerular filtration rate to $\mathrm{ml} / \mathrm{min} / 1.73 \mathrm{~m}^{2}$, divide the value by 0.01667 . 
Table 2

Changes in blood pressure components between the 2 study visits

\begin{tabular}{|l|l|l|l|}
\hline Variable $(\mathbf{n}=\mathbf{4 1 4})$ & $\begin{array}{l}\text { First study } \\
(\text { mean } \pm \text { SD })\end{array}$ & $\begin{array}{l}\text { Second study } \\
(\text { mean } \pm \text { SD })\end{array}$ & $P$-value \\
\hline Brachial SBP $(\mathrm{mmHg})$ & $134 \pm 16$ & $141 \pm 18$ & $<0.0001$ \\
\hline Brachial DBP $(\mathrm{mmHg})$ & $75 \pm 9$ & $70 \pm 10$ & $<0.0001$ \\
\hline Brachial MAP $(\mathrm{mmHg})$ & $95 \pm 10$ & $98 \pm 11$ & $<0.0001$ \\
\hline Brachial PP (mmHg) & $58 \pm 14$ & $71 \pm 17$ & $<0.0001$ \\
\hline Number of anti-hypertensive medications & $1.5 \pm 0.8$ & $2.1 \pm 1.0$ & $<0.0001$ \\
\hline Percent of participants whose blood pressure parameters increased over time \\
\hline Brachial SBP, n (\%) & $269(65 \%)$ \\
\hline Brachial DBP, n (\%) & $112(27 \%)$ \\
\hline Brachial MAP, n (\%) & $250(62 \%)$ \\
\hline Brachial PP, n (\%) & $332(80 \%)$ \\
\hline
\end{tabular}

DBP: diastolic blood pressure. MAP: mean arterial pressure. PP: pulse pressure. SBP: systolic blood pressure. 
Table 3

Independent predictors of increases in systolic, diastolic, mean and pulse pressure over time

\begin{tabular}{|c|c|c|}
\hline & $\beta \pm \mathbf{S E}$ & $P$-value \\
\hline \multicolumn{3}{|l|}{ Predictors of SBP increase } \\
\hline History of MI & $10.61 \pm 3.24$ & 0.001 \\
\hline Baseline SBP, mmHg & $-0.59 \pm 0.06$ & $<0.0001$ \\
\hline Baseline DBP, mmHg & $-0.23 \pm 0.12$ & 0.05 \\
\hline \multicolumn{3}{|l|}{ Predictors of DBP increase } \\
\hline History of smoking & $1.78 \pm 0.83$ & 0.03 \\
\hline History of MI & $4.08 \pm 1.69$ & 0.02 \\
\hline Baseline SBP, mmHg & $-0.12 \pm 0.03$ & $<0.0001$ \\
\hline Baseline DBP, mmHg & $-0.48 \pm 0.06$ & $<0.0001$ \\
\hline $\mathrm{eGFR}, \mathrm{mL} / \mathrm{min} / 1.73 \mathrm{~m}^{2}$ & $0.13 \pm 0.04$ & 0.001 \\
\hline \multicolumn{3}{|l|}{ Predictors of MAP increase } \\
\hline History of MI & $5.86 \pm 2.05$ & 0.004 \\
\hline Baseline SBP, mmHg & $-0.22 \pm 0.04$ & $<0.0001$ \\
\hline Baseline DBP, mmHg & $-0.42 \pm 0.08$ & $<0.0001$ \\
\hline Difference in the number of anti-hypertensives & $-1.39 \pm 0.63$ & 0.03 \\
\hline \multicolumn{3}{|l|}{ Predictors of PP increase } \\
\hline History of MI & $6.53 \pm 3.19$ & 0.04 \\
\hline Baseline SBP, mmHg & $-0.48 \pm 0.06$ & $<0.0001$ \\
\hline Baseline DBP, mmHg & $0.25 \pm 0.10$ & 0.02 \\
\hline
\end{tabular}

Results of linear regression models using absolute blood pressure difference between $2^{\text {nd }}$ and $1^{\text {st }}$ visits as the dependent variable. eGFR: estimated glomerular filtration rate. MI: myocardial infarction. Other abbreviations as in Table 2. 


\section{Table 4}

Associations of arterial stiffness measures with changes in blood pressure components over time

\begin{tabular}{|l|l|l|l|}
\hline $\begin{array}{l}\text { Arterial stiffness } \\
\text { measure }\end{array}$ & $\begin{array}{l}\Delta \text { blood pressure } \\
\text { component }\end{array}$ & $\beta \pm$ SE & $P$ value \\
\hline \multirow{5}{*}{ cfPWV $(\mathrm{m} / \mathrm{s})$} & $\mathrm{SBP}(\mathrm{mmHg})$ & $0.71 \pm 0.31$ & 0.02 \\
\cline { 2 - 4 } & $\mathrm{DBP}(\mathrm{mmHg})$ & $-0.37 \pm 0.18$ & 0.04 \\
\cline { 2 - 4 } & $\mathrm{MAP}(\mathrm{mmHg})$ & $-0.03 \pm 0.23$ & 0.91 \\
\cline { 2 - 4 } & $\mathrm{PP}(\mathrm{mmHg})$ & $1.09 \pm 0.27$ & $<0.0001$ \\
\hline \multirow{5}{*}{$\mathrm{AIx}(\%)$} & $\mathrm{SBP}(\mathrm{mmHg})$ & $0.23 \pm 0.10$ & 0.02 \\
\cline { 2 - 4 } & $\mathrm{DBP}(\mathrm{mmHg})$ & $0.16 \pm 0.04$ & $<0.0001$ \\
\cline { 2 - 4 } & $\mathrm{MAP}(\mathrm{mmHg})$ & $0.27 \pm 0.07$ & $<0.0001$ \\
\cline { 2 - 4 } & $\mathrm{PP}(\mathrm{mmHg})$ & $0.06 \pm 0.08$ & $\mathrm{P} 0.47$ \\
\hline \multirow{5}{*}{$\mathrm{CPP}(\mathrm{mmHg})$} & $\mathrm{SBP}(\mathrm{mmHg})$ & $0.44 \pm 0.07$ & $\mathrm{P}<.0001$ \\
\cline { 2 - 4 } & $\mathrm{DBP}(\mathrm{mmHg})$ & $0.02 \pm 0.04$ & $\mathrm{P} 0.60$ \\
\cline { 2 - 4 } & $\mathrm{MAP}(\mathrm{mmHg})$ & $0.24 \pm 0.05$ & $\mathrm{P}<0.0001$ \\
\cline { 2 - 4 } & $\mathrm{PP}(\mathrm{mmHg})$ & $0.42 \pm 0.06$ & $\mathrm{P}<0.0001$ \\
\hline
\end{tabular}

Linear regression models were adjusted for age, sex, time interval between studies, baseline SBP and DBP, history of diabetes, smoking, myocardial infarction and stroke, BMI, glomerular filtration rate, use of statins and difference in the number of anti-hypertensive medications between $2^{\text {nd }}$ and $1^{\text {st }}$ visits. We used a criteria of $\mathrm{P}<0.10$ to enter, and $\mathrm{P} \unlhd 0.05$ to stay in the models. Final covariates included in each model are shown in Table 3. AIx: aortic augmentation index. cfPWV: carotid-femoral pulse wave velocity. CPP: central pulse pressure. DBP: diastolic blood pressure. MAP: mean arterial pressure. PP: pulse pressure. SBP: systolic blood pressure. SE: standard error 
Table 5

Independent predictors of SBP, DBP, MAP and PP worsening over time

\begin{tabular}{|l|l|l|}
\hline & OR $(\mathbf{9 5 \%}$ CI) & $P$-value \\
\hline Predictors of SBP worsening & \multicolumn{2}{|l|}{} \\
\hline History of MI & $2.58(1.00,6.79)$ & 0.05 \\
\hline History of diabetes & $2.47(1.18,5.18)$ & 0.02 \\
\hline Statin use & $0.59(0.34,1.00)$ & 0.05 \\
\hline Baseline SBP, $1 \mathrm{mmHg}$ & $0.93(0.91,0.95)$ & $<0.0001$ \\
\hline Predictors of DBP worsening \\
\hline Baseline SBP, $1 \mathrm{mmHg}$ & $0.97(0.95,0.99)$ & 0.02 \\
\hline Baseline DBP, $1 \mathrm{mmHg}$ & $0.90(0.87,0.94)$ & $<0.0001$ \\
\hline Predictors of MAP worsening & $1.90(1.10,3.30)$ & 0.02 \\
\hline Female sex & $2.04(1.25,3.33)$ & 0.004 \\
\hline History of smoking & $0.96(0.94,0.97)$ & $<0.0001$ \\
\hline Baseline SBP, $1 \mathrm{mmHg}$ & $0.95(0.92,0.98)$ & 0.001 \\
\hline Baseline DBP, $1 \mathrm{mmHg}$ & \multicolumn{3}{|l}{} \\
\hline eGFR, $1 \mathrm{~mL} / \mathrm{min} / 1.73 \mathrm{~m}{ }^{2}$ & $1.03(1.01,1.05)$ & 0.001 \\
\hline Predictors of PP worsening & $1.04(1.01,1.08)$ & 0.02 \\
\hline Age, 1 year & $0.93(0.91,0.95)$ & $<0.0001$ \\
\hline Baseline SBP, $1 \mathrm{mmHg}$ & $1.04(1.01,1.08)$ & 0.02 \\
\hline Baseline DBP, $1 \mathrm{mmHg}$ &
\end{tabular}

"Worsening" was defined as a difference between blood pressure measures between the $2^{\text {nd }}$ and $1^{\text {st }}$ visits $>0$. AIx: aortic augmentation index. cfPWV: carotid-femoral pulse wave velocity. CI: confidence interval. CPP: central pulse pressure. DBP: diastolic blood pressure. MAP: mean arterial pressure. OR: odds ratio. PP: pulse pressure. SBP: systolic blood pressure. SE: standard error 